3-20-2019

\title{
Helium tune-out wavelength: Gauge invariance and retardation corrections
}

\author{
Gordon W.F. Drake \\ University of Windsor \\ Jacob Gabriel Manalo \\ University of Ottawa \\ Pei-Pei Zhang \\ Wuhan Institute of Physics and Mathematics \\ Kenneth George Herbert Baldwin \\ Australian National University
}

Follow this and additional works at: https://scholar.uwindsor.ca/physicspub

Part of the Physics Commons

\section{Recommended Citation}

Drake, Gordon W. F.; Manalo, Jacob Gabriel; Zhang, Pei-Pei; and Baldwin, Kenneth George Herbert. (2019). Helium tune-out wavelength: Gauge invariance and retardation corrections. Hyperfine Interactions.

https://scholar.uwindsor.ca/physicspub/193

This Article is brought to you for free and open access by the Department of Physics at Scholarship at UWindsor. It has been accepted for inclusion in Physics Publications by an authorized administrator of Scholarship at UWindsor. For more information, please contact scholarship@uwindsor.ca. 
Noname manuscript No.

(will be inserted by the editor)

\title{
Helium tune-out wavelength: Gauge invariance and retardation corrections
}

\author{
G. W. F. Drake, · J. G. Manalo . \\ P.-P. Zhang • K. G. H. Baldwin
}

Received: date / Accepted: date

\begin{abstract}
The problem of calculating the tune-out wavelength for an atom interacting with a plane electromagnetic wave is formulated as a zero in the Rayleigh scattering cross section, rather than a zero in the dynamic polarizability. Retardation (finite wavelength) corrections are discussed in the velocity gauge, and possible gauge transformations to a length form are investigated. For the special case of $S$-states, it is shown that a pure length form exists for the leading $p_{x} z$ retardation correction, even though one does not exist in general. The results of high-precision calculations in Hylleraas coordinates are presented for the tune-out wavelength of helium near the $2{ }^{3} S-3{ }^{3} P$ transition at $413 \mathrm{~nm}$.
\end{abstract}

Keywords tune-out wavelength $\cdot$ gauge transformation $\cdot$ retardation $\cdot$ helium

\section{Introduction}

The tune-out wavelength is the wavelength at which the interaction of an atom with an incident laser field vanishes. Recent high-precision measurements in helium [1] are sensitive to relativistic and quantum electrodynamic corrections. The effect has traditionally been formulated as a zero in the ac Stark shift for an atom trapped in an optical lattice formed by counter-propagating laser beams [2,3], and detailed relativistic configuration-interaction (CI) calculations have been carried

Gordon William Frederic Drake

Department of Physics, University of Windsor, Windsor, ON Canada N9B 3P4

E-mail: gdrake@uwindsor.ca

Jacob Gabriel Manalo

Present address: Department of Physics, University of Ottawa, Ottawa, ON K1N 6N5 Canada E-mail: manaloj@uwindsor.ca

Pei-Pei Zhang

Present address: Wuhan Institute of Physics and Mathematics, Chinese Academy of Sciences, Wuhan, Peoples Republic of China E-mail: PeiPei.Zhang@uwindsor.ca

Kenneth George Herbert Baldwin

Research School of Physics and Engineering, Australian National University, Canberra, Australian Capital Territory 0200, Australia E-mail: Kenneth.Baldwin@anu.edu.au 
out [4]. However, an optical lattice corresponds to an electric potential that is fixed in space and oscillates in time such that the interaction with an atom depends on its location relative to the nodes of the potential. Calculations are usually done in the long-wavelength approximation for the dipole and higher multipole terms generated by the optical lattice. The Baldwin experiment [1] involves instead the interaction of an atom with a single laser beam corresponding to traveling transverse plane wave with a vector potential of the form

$$
\mathbf{A}=A_{0} \hat{\boldsymbol{e}} e^{i \mathbf{k} \cdot \mathbf{r}-i \omega t}
$$

In this case, it is more appropriate to think of the tune-out wavelength as a zero in the Rayleigh scattering cross section, rather than a zero in the frequency-dependent polarizability. In other words, the tune-out wavelength is the wavelength at which the atom no longer scatters photons, and so it becomes "invisible." The ac Stark shift does not take into account the photon nature of the interaction. The two pictures are equivalent in lowest order, but not when retardation corrections are taken into account; i.e., corrections to the long wavelength approximation.

The purpose of this paper is to reformulate the tune-out wavelength as a zero in the Rayleigh scattering cross section, and to investigate its calculation in the velocity form of the interaction, as well as the length form. We also investigate the calculation of the leading retardation correction. Some of the numerical results have been previously presented [5].

\section{Rayleigh scattering formalism}

Following the derivation of Akhiezer and Berestetskii [6] from QED, the normalization factor is $e A_{0}=c e \sqrt{\hbar /(2 \omega \mathcal{V})}$, normalized to unit photon energy $\hbar \omega$ in a volume $\mathcal{V}$, the effective interaction energy is

$$
\begin{aligned}
U_{i \rightarrow f}= & \frac{2 \pi \hbar c^{2} e^{2}}{\mathcal{V} \sqrt{\omega_{i} \omega_{f}}} \sum_{n \pm}\left[\frac{\left\langle f\left|\hat{\boldsymbol{e}}_{f} e^{-i \mathbf{k}_{f} \cdot \mathbf{r}}\right| n\right\rangle\left\langle n\left|\hat{\boldsymbol{e}}_{i} e^{i \mathbf{k}_{i} \cdot \mathbf{r}}\right| i\right\rangle}{E_{n}-E_{i}-\hbar \omega_{i}}\right. \\
& \left.+\frac{\left\langle f\left|\hat{\boldsymbol{e}}_{i} e^{i \mathbf{k}_{i} \cdot \mathbf{r}}\right| n\right\rangle\left\langle n\left|\hat{\boldsymbol{e}}_{f} e^{-i \mathbf{k}_{f} \cdot \mathbf{r}}\right| i\right\rangle}{E_{n}-E_{f}+\hbar \omega_{i}}\right]
\end{aligned}
$$

summed over both positive and negative energy states. In terms of the Dirac $\gamma$ matrices, $\hat{\boldsymbol{e}}$ is defined by $\hat{\boldsymbol{e}}=\gamma_{\mu} e_{\mu}$, and $\mathbf{e}$ is the polarization vector such that $\mathbf{k} \cdot \mathbf{e}=0$. For the case of coherent Rayleigh scattering, $E_{i}=E_{f}, \mathbf{k}_{i}=\mathbf{k}_{f}$, and $\omega_{i}=\omega_{f}=\omega$. Let us assume for the sake of definiteness that the propagation vector $\mathbf{k}$ points in the $z$-direction, and the wave is plane-polarized in the $x$-direction. (The results are easily generalized to the case of arbitrary directions of propagation and polarization.) The expression for $U_{i \rightarrow f}$ then becomes

$U=\frac{2 \pi c^{2} e^{2} \hbar}{\omega \mathcal{V}} \sum_{n \pm}\left[\frac{\left\langle i\left|\alpha_{x} e^{-i k z}\right| n\right\rangle\left\langle n\left|\alpha_{x} e^{i k z}\right| i\right\rangle}{E_{n}-E_{i}-\hbar \omega}+\frac{\left\langle i\left|\alpha_{x} e^{i k z}\right| n\right\rangle\left\langle n\left|\alpha_{x} e^{-i k z}\right| i\right\rangle}{E_{n}-E_{i}+\hbar \omega}\right]$

where $k=\omega / c$. For convenience, let us write this in the form

$$
U=\frac{2 \pi c^{2} e^{2} \hbar}{\omega \mathcal{V}} \sum_{n \pm}\left|\left\langle i\left|\alpha_{x} e^{i k z}\right| n\right\rangle\right|^{2}\left(\frac{1}{\Delta E_{n}+\bar{\omega}}+\frac{1}{\Delta E_{n}-\bar{\omega}}\right)
$$


where $\Delta E_{n}=E_{n}-E_{i}$ and $\bar{\omega}=\hbar \omega$. For a many-electron atom, the transition operator $\alpha_{x} e^{i k z}$ is understood to be summed over the $N$ atomic electrons.

The contribution from negative energy states in Eq. (4) makes an important contribution, even in the nonrelativistic limit. It could be handled directly without approximation by introducing a complete basis set of relativistic Sturmian functions [7-9]. However, our aim here is to obtain equivalent nonrelativistic operators. As discussed by Akhiezer and Berestetskii [6], in the nonrelativistic limit $\hbar \omega<<m c^{2}$, the denominators in Eq. (4) can be replaced by a constant $E_{n} \simeq-2 m c^{2}$. The sum over $n^{-}$can then be extended to a sum over all $n^{ \pm}$with the introduction of negative energy projection operators, and the sum completed by closure. In this approximation, the contribution from negative energy states is proportional to the matrix element $\left\langle f\left|\mathbf{e}_{i} \cdot \mathbf{e}_{f} \exp \left[i\left(\mathbf{k}_{i}-\mathbf{k}_{f}\right) \cdot \mathbf{r}\right]\right| i\right\rangle$, which just reduces to $N \delta_{i f}$ for the case of coherent scattering with $\mathbf{k}_{f}=\mathbf{k}_{i}$, and $N$ is the number of electrons. Then $U^{-}$becomes

$$
\begin{aligned}
U^{-} & =-\frac{2 N \pi c^{2} e^{2} \hbar}{\omega \mathcal{V}}\left(\frac{1}{2 m c^{2}+\bar{\omega}}+\frac{1}{2 m c^{2}-\bar{\omega}}\right) \\
& \simeq-\frac{2 N \pi e^{2} \hbar}{m \omega \mathcal{V}}\left[1+\left(\frac{\bar{\omega}}{2 m c^{2}}\right)^{2}+\cdots\right]
\end{aligned}
$$

There are of course other binding-energy corrections, which are known in the oneelectron case from Lamb shift calculations [10], but the second term in Eq. (5) is certainly one of the frequency-dependent terms that should be included.

$U^{-}$can now be combined with the remaining sum over positive energies to obtain the total

$$
\begin{aligned}
U= & \frac{2 \pi c^{2} e^{2} \hbar}{\omega \mathcal{V}}\left\{\sum_{n^{+}}\left|\left\langle i\left|\alpha_{x} e^{i k z}\right| n\right\rangle\right|^{2}\left(\frac{1}{\Delta E_{n}+\bar{\omega}}+\frac{1}{\Delta E_{n}-\bar{\omega}}\right)\right. \\
& \left.-\frac{N}{m c^{2}}\left[1+\left(\frac{\hbar \omega}{2 m c^{2}}\right)^{2}+\cdots\right]\right\}
\end{aligned}
$$

The usual procedure at this point is to make the long wavelength approximation and assume that $k z<<1$ for values of $z$ within the dimensions of the atom, and to insert the retardation expansion

$$
e^{i k z}=1+i k z-\frac{1}{2}(k z)^{2}+\cdots
$$

This is rapidly convergent for nonrelativistic frequencies $\omega=k / c$, and so only the leading three terms will be considered for now.

Let us first consider the nonretarded limit where only the first term of the righthand-side of Eq. (7) is kept. In that case, the transition matrix element is simply $\left\langle i\left|\alpha_{x}\right| n\right\rangle$, evaluated in terms of Dirac wave functions. In the nonrelativistic limit, we can make the replacement

$$
\alpha \cdot \mathbf{A} \rightarrow \frac{\mathbf{p} \cdot \mathbf{A}}{m c}+\boldsymbol{\mu} \times \mathbf{A}
$$

where $\boldsymbol{\mu}=(e \hbar / 2 m c) \boldsymbol{\sigma}$ is the spin magnetic moment of the electron. Neglecting for now the spin-dependent part, Eq. (6) then simplifies to

$$
U=\frac{2 \pi e^{2} \hbar}{\omega m \mathcal{V}}\left\{\frac{1}{m} \sum_{n^{+}}\left|\left\langle i\left|p_{x}\right| n\right\rangle\right|^{2}\left(\frac{1}{\Delta E_{n}+\bar{\omega}}+\frac{1}{\Delta E_{n}-\bar{\omega}}\right)-N\right\}
$$


Equation (9) corresponds to the "velocity" (or momentum) form of the interaction. Calculations of tune-out wavelengths are typically done in the equivalent "length" form involving matrix elements of $x$ in place of $p_{x}$. The transformation can be accomplished by making use of the commutation relation

$$
[H, x]=\frac{i \hbar}{m} p_{x}
$$

together with $\left(E_{n}-E_{i}\right)\langle i|x| n\rangle=-\langle n|[H, x]| i\rangle$, where $x$ is a short-hand notation for $\sum_{k=1}^{N} x_{k}$. An interesting feature is that the summation term in Eq. (9) tends to $N$ in the limit $\omega \rightarrow 0$ (analagous to the Thomas-Reiche-Kuhn oscillator strength sum rule) and so the two terms cancel. To see this more clearly, it is useful to expand the energy denominators in the form

$$
\frac{1}{\Delta E_{n}+\bar{\omega}}+\frac{1}{\Delta E_{n}-\bar{\omega}}=\frac{2}{\Delta E_{n}}\left(1+\frac{\bar{\omega}^{2}}{\Delta E_{n}^{2}}+\cdots\right)
$$

and correspondingly expand $U=U^{(0)}+U^{(2)}+\cdots$. The leading two terms are then

$$
U^{(0)}=\frac{2 \pi e^{2} \hbar}{\omega m \mathcal{V}}\{N-N\}=0
$$

and

$$
U^{(2)}+\cdots=\frac{2 \pi e^{2} \hbar}{\omega m \mathcal{V}}\left\{2 \omega^{2} m \sum_{n^{+}} \frac{\langle i|x| n\rangle\langle n|x| i\rangle}{\Delta E_{n}}\left(1+\frac{\bar{\omega}^{2}}{\Delta E_{n}^{2}}+\cdots\right)\right\}
$$

The infinite series in $\left(\omega / \Delta E_{n}\right)^{2}$ can then be resummed to infinity to obtain the final length form

$$
\begin{aligned}
U & =\frac{2 \pi e^{2} \bar{\omega}}{\mathcal{V}} \sum_{n^{+}}\langle i|x| n\rangle\langle n|x| i\rangle\left(\frac{1}{\Delta E_{n}+\bar{\omega}}+\frac{1}{\Delta E_{n}-\bar{\omega}}\right) \\
& \equiv \frac{2 \pi \bar{\omega}}{\mathcal{V}} \alpha_{d}(\omega)
\end{aligned}
$$

where $\alpha_{d}(\omega)$ is the frequency-dependent dipole polarizability. At this level, it makes no difference whether one identifies the tune-out wavelength as a zero in $\alpha_{d}(\omega)$, or a zero in the effective interaction energy $U(\omega)$ responsible for Rayleigh scattering. However, it does make a difference when retardation corrections are considered, as discussed in the following section. There is a further qualitative difference in the interaction energy in limit $\omega \rightarrow 0$. In this limit, $U(\omega)$ vanishes, but the static dipole polarizability $\alpha_{d}$ remains constant.

\section{Retardation Corrections}

Retardation (finite wavelength) corrections to the results in the previous section come from the higher-order terms in Eq. (7). They must be calculated in the velocity form of the interaction because, in general, a length form as usually defined does not exist beyond the lowest-order term for any given multipole [11]. The following paragraphs provide a rationale for the nonexistence of a simple length form. 
According to the usual argument, the equivalence of the length and velocity forms can be viewed as a gauge transformation of the electromagnetic fields $E$ and $B$ which satisfy Maxwell's equations

$$
\begin{aligned}
& \mathbf{E}=-\nabla V-\frac{1}{c} \frac{\partial \mathbf{A}}{\partial t} \\
& \mathbf{B}=\nabla \times \mathbf{A}
\end{aligned}
$$

The physical fields $\mathbf{E}$ and $\mathbf{B}$ are invariant under an arbitrary transformation of the scalar and vector potentials $V$ and $A$ according to

$$
\begin{aligned}
& \mathbf{A} \longrightarrow \mathbf{A}+\nabla f(\mathbf{r}, t) \\
& V \longrightarrow V-\frac{1}{c} \frac{\partial f(\mathbf{r}, t)}{\partial t}
\end{aligned}
$$

where $f(\mathbf{r}, t)$ is an arbitrary differentiable function of $\mathbf{r}$ and $t$. For a wave propagating in the $z$-direction and polarized in the $x$-direction, choose

$$
\begin{aligned}
\mathbf{A} & =A_{0} \hat{\mathbf{e}}_{\mathbf{x}} e^{i k z-i \omega t} \\
f(\mathbf{r}, t) & =\mathcal{C} e^{i k z-i \omega t}
\end{aligned}
$$

where $\mathcal{C}$ is an arbitrary constant. Then the gauge transformation yields

$$
\begin{aligned}
\mathbf{A} & \rightarrow A_{0} \hat{\mathbf{e}}_{\mathbf{x}} e^{i k z-i \omega t}+\mathcal{C} i k \hat{\mathbf{e}}_{\mathbf{z}} e^{i k z-i \omega t} \\
V & \rightarrow V+\mathcal{C} \frac{i \omega}{c} e^{i k z-i \omega t}
\end{aligned}
$$

where $k=\omega / c$. Define a new arbitrary constant $\mathcal{C}^{\prime}=i k \mathcal{C}$. Then

$$
\begin{aligned}
& \mathbf{A}=A_{0} \hat{\mathbf{e}}_{\mathbf{x}} e^{i k z-i \omega t}+\mathcal{C}^{\prime} \hat{\mathbf{e}}_{\mathbf{z}} e^{i k z-i \omega t} \\
& V=V_{0}+\mathcal{C}^{\prime} e^{i k z-i \omega t}
\end{aligned}
$$

Thus the gauge transformation introduces a longitudinally polarized component $\mathcal{C}^{\prime} \hat{\mathbf{e}}_{\mathbf{z}} e^{i k z-i \omega t}$ counterbalanced by an additional contribution $\mathcal{C}^{\prime} e^{i k z-i \omega t}$ to the scalar potential $V$, while leaving the physical $\mathbf{E}$ and $\mathbf{B}$ fields unchanged.

Quantum mechanical matrix elements are also unchanged, provided that the wave functions are exact, and this leads to the "length" form of the transition operator, at least in lowest order, as follows. Equating matrix elements of the $\mathcal{C}^{\prime}$ dependent parts of $-(\mathbf{p} \cdot \mathbf{A}+\mathbf{A}) \cdot \mathbf{p} /(2 m c)$ and $V$ between arbitrary orthogonal states $|a\rangle$ and $|b\rangle$ yields

$$
\frac{1}{2 m c}\left\langle a\left|p_{z} e^{i k z}+e^{i k z} p_{z}\right| b\right\rangle=-\left\langle a\left|e^{i k z}\right| b\right\rangle
$$

where, by conservation of energy, $k=\omega_{a b} / c$. This equation is satisfied term-byterm to all orders in the expansion $e^{i k z}=1+i k z+\cdots$, provided that the wave functions are exact and orthogonal. This can easily be seen by making the substitution

$$
\begin{aligned}
\left\langle a\left|(i k z)^{n+1}\right| b\right\rangle & =i \omega_{a b} \frac{(i k)^{n}}{c}\left\langle a\left|z^{n}\right| b\right\rangle \\
& =i \frac{(i k)^{n}}{\hbar c}\left\langle a\left|\left[H_{0}, z^{n}\right]\right| b\right\rangle
\end{aligned}
$$


and evaluating the commutator with the field-free Hamiltonian $H_{0}$. The leading nonvanishing terms give

$$
\frac{1}{m c}\left\langle a\left|p_{z}\right| b\right\rangle=-\frac{i \omega_{a b}}{c}\langle a|z| b\rangle
$$

This is the usual equivalence of the length and velocity forms. However, the correct velocity operator for our assumed plane wave is $\boldsymbol{p}_{\boldsymbol{x}} e^{\boldsymbol{i k z}}$, not $\boldsymbol{p}_{\boldsymbol{z}} e^{\boldsymbol{i k \boldsymbol { z }}}$. In general, there is no equivalent length form valid beyond leading order [11].

3.1 Gauge invariance of multipole expansions

A similar argument applies to each partial wave if the plane wave discussed so far with definite direction of propagation $\mathbf{k}$ and direction of polarization $\hat{\mathbf{e}}$ in terms of definite angular momentum $L$ and parity. It is always possible to write

$$
\bar{\omega}_{a b}\left\langle a\left|r^{L} Y_{L}^{M}\right| b\right\rangle=\left\langle a\left|\left[H, r^{L} Y_{L}^{M}\right]\right| b\right\rangle
$$

where $Y_{L}^{M}$ is a spherical harmonic. Furthermore, since $\nabla^{2} r^{L} Y_{L}^{M}(\theta, \phi)=0$, it follows that (in atomic units)

$$
\begin{aligned}
{\left[H, r^{L} Y_{L}^{M}\right] } & =-\frac{1}{2}\left[\nabla^{2}, r^{L} Y_{L}^{M}\right] \\
& =-\nabla r^{L} Y_{L}^{M} \cdot \nabla \\
& =-i \nabla r^{L} Y_{L}^{M} \cdot \mathbf{p}
\end{aligned}
$$

which is the correct velocity form of the operator. However, this works only to lowest order. The function $r^{L}$ is just the leading term in the power series expansion of the spherical Bessel function $j_{L}(k r)=j_{L}(\omega r / c)$ [14], and in general $\nabla^{2} r^{K} Y_{L}^{M}(\theta, \phi) \neq 0$ unless $K=L$.

\subsection{The leading retardation term}

As an illustrative example, the operator for the leading retardation correction to the velocity form $p_{x} e^{i k z}$ is $i k p_{x} z$. In general, there is no corresponding pure length form [11], but for the special case of the interaction energy $U$ for spherically symmetric $S$-states, the operator can be symmetrized with respect to $x$ and $z$ to obtain, for matrix elements

$$
\begin{aligned}
\left\langle a\left|\frac{i k}{2 m c}\left(p_{z} x+p_{x} z\right)\right| b\right\rangle & =-\frac{k}{2 \hbar c}\left\langle a\left|\left[H_{0}, x z\right]\right| b\right\rangle \\
& =-\frac{k^{2}}{2}\langle a|x z| b\rangle
\end{aligned}
$$

evaluated at $k=\omega_{a b} / c$. The right-hand-side looks superficially like a quadrupole interaction, but in fact it is a retardation correction. It is a factor of two smaller 
than what one might naively expect. Using this equivalence, the velocity and length forms of the $x z$-retardation correction are

$$
\begin{aligned}
& \Delta U_{x z}^{V}=\frac{2 \pi e^{2} \hbar}{\omega m \mathcal{V}}\left\{\frac{k^{2}}{m} \sum_{n^{+}}\left|\left\langle i\left|p_{x} z\right| n\right\rangle\right|^{2}\left(\frac{1}{\Delta E_{n}+\bar{\omega}}+\frac{1}{\Delta E_{n}-\bar{\omega}}\right)\right\} \\
& \Delta U_{x z}^{L}=\frac{2 \pi e^{2} \bar{\omega}}{\mathcal{V}}\left\{\frac{k^{2}}{4} \sum_{n^{+}}|\langle i|x z| n\rangle|^{2}\left(\frac{1}{\Delta E_{n}+\bar{\omega}}+\frac{1}{\Delta E_{n}-\bar{\omega}}\right) \frac{\Delta E_{n}^{2}}{\bar{\omega}^{2}}\right\}
\end{aligned}
$$

The results presented in the following section demonstrate that the above expressions yield identical results to within the accuracy allowed by the variational wave functions. The next $(k z)^{2} / 2$ in Eq. (7) also contributes as a cross-term after squaring. This and other finite mass and relativistic corrections will be discussed separately in a future publication.

\section{Calculations and Conclusions}

This section presents results of calculations of the tune-out wavelength for helium near the $413 \mathrm{~nm}$ transition corresponding to the $2{ }^{3} S-3{ }^{3} \mathrm{P}$ transition. The fieldfree Hamiltonian for helium in reduced mass atomic units is

$$
H_{0}=-\frac{1}{2} \nabla_{1}^{2}-\frac{1}{2} \nabla_{2}^{2}-\frac{Z}{r_{1}}-\frac{Z}{r_{2}}+\frac{1}{r_{12}}-\frac{\mu}{M} \nabla_{1} \cdot \nabla_{2}
$$

where the last term is the mass polarization term, and $\mu$ is the electron reduced mass. The transition matrix elements and energies were evaluated in terms of variational solutions to the Schrödinger equation with wave functions expressed in Hylleraas coordinates of the form $[15,16]$

$$
\Psi\left(\mathbf{r}_{1}, \mathbf{r}_{2}\right)=\sum_{p=1}^{2} \sum_{i, j, k} a_{i j k}^{(p)} r_{1}^{i} r_{2}^{j} r_{12}^{k} e^{-\alpha_{p} r_{1}-\beta_{p} r_{2}} \mathcal{Y}_{l_{1} l_{2} L}^{M}\left(\hat{\mathbf{r}}_{\mathbf{1}}, \hat{\mathbf{r}}_{\mathbf{2}}\right) \pm \mathbf{1} \leftrightarrow \mathbf{2}
$$

with $i+j+k \leq \Omega, \quad \Omega=4,5,6, \cdots 15$, and $\mathcal{Y}_{l_{1} l_{2} L}^{M}\left(\hat{\mathbf{r}}_{\mathbf{1}}, \hat{\mathbf{r}}_{\mathbf{2}}\right)$ is a vector-coupled product of spherical harmonics for the two electrons to form a state of total angular momentum $L$ and component $M$. The sum over $p$ allows for two (or more) individually optimized distance scales as determined by the nonlinear parameters $\alpha_{p}$ and $\beta_{p}$. The resulting wave functions have exceptional numerical stability so that standard quadruple precision arithmetic (approximately 32 decimal digits) is sufficient.

The infinite sums over intermediate states in the previous section (including an integration over the continua) were replaced by finite sums over variationally determined pseudostates obtained by diagonalizing the Hamiltonian in an $N$-dimensional basis set of $P$-states for the nonrelativistic tune-out wavelength, and $D$-states for the $x z$-retardation correction.

As an illustrative example, Table 1 shows the convergence with basis set size for the nonrelativistic tune-out wavelength $\lambda_{t}$ in both the length $(\mathrm{L})$ and velocity (V) forms. It is significant that the velocity form is more rapidly convergent with basis set size (as controlled by $\Omega$ ) by more than an order of magnitude, even though it does not yield an accurate value for the static polarizability $\alpha_{d}$ due to 
Table 1 Nonrelativistic tune-out wavelength $\lambda_{t}$ near the $2{ }^{3} S-3{ }^{3} P$ transition of helium at $413 \mathrm{~nm}$ for the case of infinite nuclear mass, calculated in both the length (L) and velocity (V) forms. $N_{S}$ and $N_{P}$ are the numbers of terms in the $S$ - and $P$-state basis sets respectively for each $\Omega=(i+j+k)_{\max } . \Delta \lambda_{t}^{L}$ and $\Delta \lambda_{t}^{V}$ are the changes in $\lambda_{t}^{L}$ and $\lambda_{t}^{V}$ for successive values of $\Omega$ to show the respective rates of convergence.

\begin{tabular}{rrrccr}
\hline \hline$\Omega$ & $N_{S} / N_{P}$ & $\lambda_{t}^{L}(\mathrm{~nm})$ & $\Delta \lambda_{t}^{L}$ & $\lambda_{t}^{V}(\mathrm{~nm})$ & \multicolumn{1}{c}{$\Delta \lambda_{t}^{V}$} \\
\hline 4 & $35 / 104$ & 413.1406819343 & & 413.0316829604 & \\
5 & $56 / 164$ & 413.0468128503 & -0.0938690840 & 413.0367383288 & 0.0050553684 \\
6 & $84 / 238$ & 413.0401876300 & -0.0066252203 & 413.0381115461 & 0.0013732173 \\
7 & $120 / 300$ & 413.0386567622 & -0.0015308678 & 413.0382787252 & 0.0001671791 \\
8 & $164 / 371$ & 413.0383943741 & -0.0002623881 & 413.0382950253 & 0.0000163001 \\
9 & $218 / 457$ & 413.0383453450 & -0.0000490291 & 413.0383016088 & 0.0000065835 \\
10 & $281 / 551$ & 413.0383087062 & -0.0000366388 & 413.0383040087 & 0.0000023999 \\
11 & $356 / 660$ & 413.0383054303 & -0.0000032759 & 413.0383043265 & 0.0000003178 \\
12 & $441 / 775$ & 413.0383045622 & -0.0000008681 & 413.0383043887 & 0.0000000622 \\
13 & $540 / 905$ & 413.0383044523 & -0.0000001099 & 413.0383043817 & -0.0000000070 \\
14 & $650 / 1039$ & 413.0383043970 & -0.0000000553 & 413.0383043859 & 0.0000000042 \\
15 & $776 / 1189$ & $413.0383043893(35)$ & -0.0000000077 & $413.0383043858(1)$ & -0.0000000001 \\
Other theory & $413.03828(3)^{\mathrm{a}}$ & & & \\
\hline \hline
\end{tabular}

numerical cancellation (cf. Eq. (12).) Also, the length form tends to converge from above while the velocity form converges (predominantly) from below. The result for the finite nuclear mass case for ${ }^{4} \mathrm{He}$ with $\mu / M=1.370745665 \times 10^{-4}$ is

$$
\lambda_{t}\left({ }^{4} \mathrm{He}\right)=413.0825905833 m_{e} / \mu=413.1392214630(1) \mathrm{nm}
$$

where $\mu / m_{e}=1-\mu / M$ is the reduced mass to electron mass ratio, and for the finite nuclear mass case, the factor of $1 / m$ in Eq. (9) should be replaced by $(1 / m)(1+$ $\mu / M)$. The numerical result marginally disagrees with the nonrelativistic value 413.139 19(2) nm from Ref. [4].

Finally, the calculated $x z$-retardation correction is $\delta \lambda_{t}^{(x z)}=0.0005600236 \mathrm{~nm}$ assuming infinite nuclear mass. This is a correction of nominal order $\alpha^{2}$, and so is significant relative to other relativistic and QED corrections. It has not been included in previous calculations. A detailed discussion of these other corrections will be given in a future publication. They must also be included before a meaningful comparison can be made with the experimental value $\lambda_{t}=413.0938\left(9_{\text {stat }}\right)\left(\left(20_{\text {syst }}\right)\right.$ [1], and the most recent theoretical value $\lambda_{t}=413.09015(4) \mathrm{nm}$ [17].

In conclusion, we have reformulated the tune-out wavelength problem as a zero in the Rayleigh scattering cross section instead of a zero in the frequencydependent polarizability, and demonstrated how the calculation can be done in the velocity form as well as the length form. The velocity form turns out to be more rapidly convergent for the tune-out wavelength, but not for the static polarizability. In addition, we have shown how retardation corrections can be calculated in the velocity form, even though a pure length form in general does not exist. The choice of which formalism to use depends entirely on the experimental conditions, and in particular whether the atom is interacting with a travelling wave as expressed by Eq. (1) or a standing wave in an optical lattice. In future work, relativistic and QED corrections will be discussed, including corrections to the radiative transition operator [18]. 


\section{Aclnowledgments}

We are grateful to Li-Yan Tang for helpful correspondence concerning her calculations. Research support by the Natural Sciences and Engineering Research Council, by SHARCnet, and by the Australian Research Council Discovery Project DP180101093 are gratefully acknowledged. P.-P. Zhang acknowledges support by the National Natural Science Foundation of China under Grant No. 11604369, and the Strategic Priority Research Program of the Chinese Academy of Sciences under Grants No. XDB21010400.

\section{References}

1. B. M. Henson, R. I. Khakimov, R. G. Dall, K. G. H. Baldwin, L. Y. Tang, and A. G. Truscott, Phys. Rev. Lett. 115, 043004 (2015).

2. M. Šindelka, N. Moiseyev, and L. S. Cederbaum, Phys. Rev. A 74, 053420 (2006).

3. U. D. Jentschura and C. M. Adhikari, Phys. Rev. A 97, 062120 (2018).

4. Y.-H. Zhang, L.-Y. Tang, X.-Z. Zhang, and T.-Y. Shi, Phys. Rev. A 93, 052516 (2016).

5. Jacob Manalo, M.Sc. Thesis, University of Windsor, Canada (2017), https://scholar.uwindsor.ca/etd/7275/

6. A. I. Akhiezer and V.B. Berestetskii, Quantum Electrodynamics, translated by G.M. Volkoff (Interscience, New York. 1965), pp. 484-491.

7. S. P. Goldman and G.W.F. Drake, Phys. Rev. A 24, 183 (1981).

8. G. W. F. Drake and S.P. Goldman, Relativistic Sturmian and finite basis set methods in atomic physics, Adv. At. Mol. Phys. 25, 393-416 (1988).

9. R. Szmytkowski, Phys. Rev. A 65, 012503 (2001), and earlier references therein.

10. K. Pachucki, Phys. Rev. A 46, 648 (1992).

11. Although a pure length form does not exist, Selst $\varnothing$ and Føorre [12] have obtained a hybrid length/velocity form by means of a unitary transformation. It can be obtained from the results presented here by calculating the commutator $\left[H_{0}, x e^{i k z-i \omega t}\right]$ and subtracting out the spurious terms generated by the commutator to obtain the correct velocity form $p_{x} e^{i k z-i \omega t}$.

12. S. Selst $\varnothing$ and M. Førre, Phys. Rev. A 76, 023427 (2007). See also Ref. [13].

13. A. D. Bandrauk, F. Fillion-Gourdeau and E. Lorin, J. Phys. B 46, 153001 (2013), Sect. 4.3 .

14. Ref. [6], Sect. 27.2

15. E. A. Hylleraas, Z. Phys. 48, 469 (1928); 54, 347 (1929).

16. G. W. F. Drake and Z.-C. Yan, Phys. Rev. A 46, 2378 (1992).

17. Y.-H. Zhang, F.-F. Wu,P.-P. Zhang, L.-Y. Tang, J.-Y. Zhang, K. G. H. Baldwin, and T.-Y. Shi, private communication (2018).

18. K. Pachucki, Phys. Rev. A 67, 012504 (2003); 69, 052502 (2004). 